\title{
Effects of Base Support Changes during Implicit Condition on Standing Postural Strategy
}

\author{
Nur Fatin Fatina bt. Mohd Ramli, Ogawa Sho, and Yamamoto Shin-ichiroh \\ Shibaura Institute of Technology, Graduate School of Engineering and Science, Saitama, Japan \\ Email: \{nb16106, mf17015, yamashin\}@ shibaura-it.ac.jp
}

\begin{abstract}
Hip and ankle strategies are commonly used in order to maintain and regain balance when people felt the risk of falling, especially in older people. The selection of postural responses was influenced by many factors such as perturbation conditions, experience, adaptation and also fear of falling. Previous studied had reported that the postural coordinate patterns had changed with translation frequency. However, since there is a possibility that the translation surface may be perceived visually and aurally, the changed of inclined surface during dynamic translation perturbation were considered. Thus, the main objective of this paper is to investigate the standing postural strategy during explicit (presence) and implicit (absence) condition of perception sensory at inclined support surface translation with different type of perturbation and sensory sense. Comparison with frequency and postural coordinate patterns under these conditions were observed. Six healthy adults had participated in this experiment study. The subjects were asked to maintain their balance and the standing postural control with and without the presence sensation during inclined support surface translation were observed. The trunk and head were fixed at the fast frequency. Furthermore, muscle activities were active during the implicit condition than the explicit condition. Furthermore, without the visual information, the body intended to sway more and higher activation muscle was observed. This study concluded that the influence from the upper center of the body due to the existences of perception sensory affects the postural control strategy. Besides, the explicit of perception sensory and visual information was important in maintaining our balance. For future investigation, consideration of the existence of perception sensory with translation surface changes is useful for fall prevention.
\end{abstract}

Index Terms-perturbation, inclined translation, base support changes, postural coordination

\section{INTRODUCTION}

Millions of people spend a good deal of their time on their feet for standing, walking or running. D.A. Winter (1995) had stated that human is inherently unstable due to large body mass is located high above a relatively small base of support. There are three major sensory systems that involved in balance and posture such as vision (planning motion and avoiding obstacles along the way), vestibular (detect linear and angular acceleration) and

Manuscript received April 26, 2017; revised June 26, 2017 somatosensory/proprioceptive systems (sense the position, velocity, orientation of gravity and contact with external objects) [1]. Sufficient feedback information from these senses can make the balancing process become effective.

In recent years, physical strength and athletic ability have been declined due to economic growth, eating habits, and lack of exercises. Additionally, certain health condition, injuries, diseases such as stroke and also aging have become the factors for the balance disorder. Balance disorder will cause dizziness, falling, floating sensation, blur vision and most importantly, it will affect standing ability [2], [3]. Focusing on the elderly, the degeneration of the balance control systems becomes the main reason of the high risk of fall and it has forced the researchers and clinicians to investigate and understanding more about how the system works [1]. Along with increasing ages, decrease in balancing function becomes one factor in falling for elderly person. Therefore, by considering the postural control mechanism, it will be useful for falling prevention.

The selection of postural responses was influenced by the perturbation conditions, experience, adaptation and also fear of falling [4]-[6]. However, the selecting of an ankle and hip strategy in postural control balance still remain unclear. The capability of accelerating center of mass (COM) can be increased by using hip strategy and the muscle activity for the desired COM acceleration is lower during hip strategy [7]. Besides, the selection of a specific postural response can be explained by the balance between energy consumption and postural stability [8].

Many researchers have been reported on factors that influence postural control strategy. Sasagawa et al. have reported that the musculoskeletal systems and nervous systems change with the inclination of the support surface translation [9]. In addition, Buchanan et al. have conducted experiment to examine the effect of frequency in sinusoidal platform translation on postural movement. The coordination patterns between body segments were changed due to the changes of frequency was reported [10]. Therefore, the perturbation type and frequency become important factors in investigating postural control strategies. Besides, Ishizawa et al. and Kawano et al. had further the investigation by changing the angle of the platform translation frequency [11], [12]. However, due to the possibility that the surface support inclination may be perceived visually and aurally, changes of the support 
surface inclination during dynamic perturbation was considered.

Thus, this paper main objective is to investigate the effect of perception in inclined support surface and the influence of postural systems strategy with the changes of platform angle and frequency.

\section{METHODS}

\section{A. Subject Preparation}

In this study, six healthy young male subjects had participated (age: $21 \pm 0.7$ [year], height $172 \pm 4.3[\mathrm{~cm}$, weight $61 \pm 7.2[\mathrm{~kg}])$. Each subject was given an explanation regarding the procedure of this experiment. None of the subjects had a history or clinical evidence of neurological, musculoskeletal or other medical conditions.

\section{B. Apparatus}

For the experiment equipment, a 6-axis movable platform (MB-150, Cosmate, Japan) was used as a platform for introducing surface type of perturbation for the subject. Both platform motion and displacement and frequency can be regulated. A motion capture system with seven high-precision infrared cameras (HWK200RT camera, Motion Analysis, USA), force plate (9286A, Kitsler, Japan) was used for recording posture motion changes during the experiment. Each device connected to $\mathrm{A} / \mathrm{D}$ converter.

\section{Experimental Protocol and Data Recording}

The subjects were instructed to stand upright on the motion base platform attached with force plate where external perturbation applied. With both hands were crossing they required to maintain their balance position for a specific period. During the experiment session, posture motion and muscle activities were recorded simultaneously. Additional trials were conducted in which case of subject was almost felt or occasional steps occurred.

The subjects were exposed to three types of sine wave external perturbation, which were TD: Toes Down, LV: Level and TU: Toes Up at two different frequencies $(0.2$ and $0.8 \mathrm{~Hz}$ ) produced by a movable platform (Fig. 1). For TU and TD condition, angle of movable platform was changed with 1.5 [deg.], 2.0 [deg.] and 2.5 [deg.] before and in between the perturbation in order to observe the changes of standing postural strategy towards the inclined surface during the explicit and implicit condition. Furthermore, the subjects' vision (eyes open (EO) and eyes close (EC)) was manipulated. All three different perturbations were delivered in a random sequence.

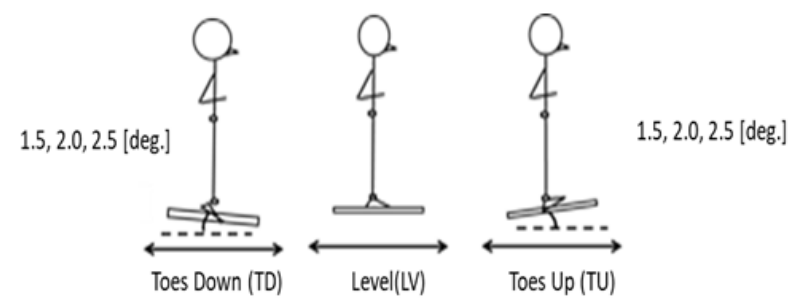

Figure 1. Definition of inclined support surface in Toes Down (TD), Level (LV) and Toes Up (TU) and condition.
There are few settings were implemented for subject preparation. These included number of reflective markers used for body motion recording using a motion capture system. In this study a fix of 17 reflective markers (placed at $3^{\text {rd }}$ metatarsal, lateral malleolus, lateral condyle, trochanter of the femur, iliac crest, acromion of spacula and top of head) were attached on subject's joints. Besides, during all experiment trials, both right and left knee joints locked using wood splints to prevent bias movement at the knees.

Furthermore, subject body also was attached with electromyography (EMG) electrodes to capture muscle activities while the subject was tried to maintain their position on the moving platform. The electrode was positioned at four different muscles at lower extremities which are bicep femoris (BF), rectus femoris (RF), tibia anterior (TA) and medial gastrocnemius (mGas) and were recorded at a sampling frequency of $1 \mathrm{kHz}$. Besides, kinematic data were obtained from a motion capture system. The sampling frequency of the motion capture system was $200 \mathrm{~Hz}$.

\section{Data Analysis}

Measured data were collected in terms of center of pressure (COP), center of mass and muscle activations. The raw data from the force plate and EMG were filtered by a second-order Butterworth filter with a $6 \mathrm{~Hz}$ cut-off to eliminate noise especially from power line and movement. A comparison of kinematics data and EMG data during explicit and implicit condition towards the inclined support surface were made using statistical analysis (mean and standard deviation); and the difference between the support surface type conditions, and comparison toward visual information were compared using two-way analyses of variance (ANOVA) with a significant level of $p<0.05$. All statistical analyses were completed using the MATLAB software.

\section{RESUlTS AND DISCUSSIONS}

Fig. 2 shows one cycle stick figure under two different frequencies at level condition. Fig. 3 below shows the head trajectory average and movable platform signal average at $0.2 \mathrm{~Hz}$ and $0.8 \mathrm{~Hz}$ frequencies. In low perturbation translation $(0.2 \mathrm{~Hz})$, subjects rode the platform with little damping at head and trunk followed the anterior-posterior motion. However, at the fast perturbation translation $(0.8 \mathrm{H})$, subjects fix their head and trunk motion in order to maintain balance. In Fig. 3 the amplitude of head motion was decreased with the increasing of the frequency.

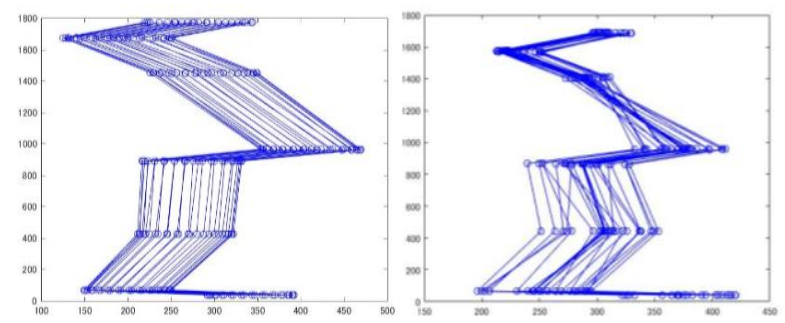

Figure 2. Stick figure under two different frequencies (left: $0.2 \mathrm{~Hz}$, right: $0.8 \mathrm{~Hz})$. 


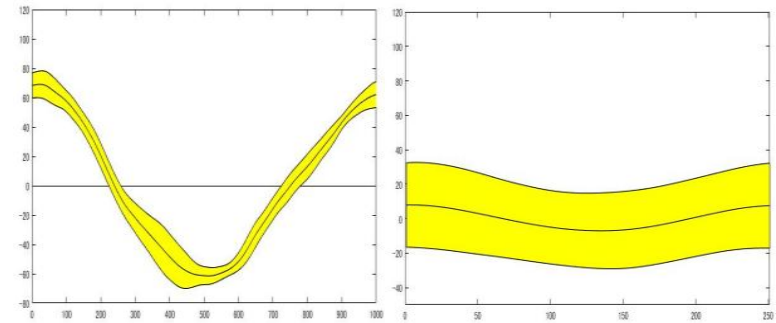

Figure 3. Head amplitude with frequency changes (left: $0.2 \mathrm{~Hz}$, right: $0.8 \mathrm{~Hz}$ ). Head amplitude is shown by the use of solid line. Shaded area around the average amplitude represents standard deviation.
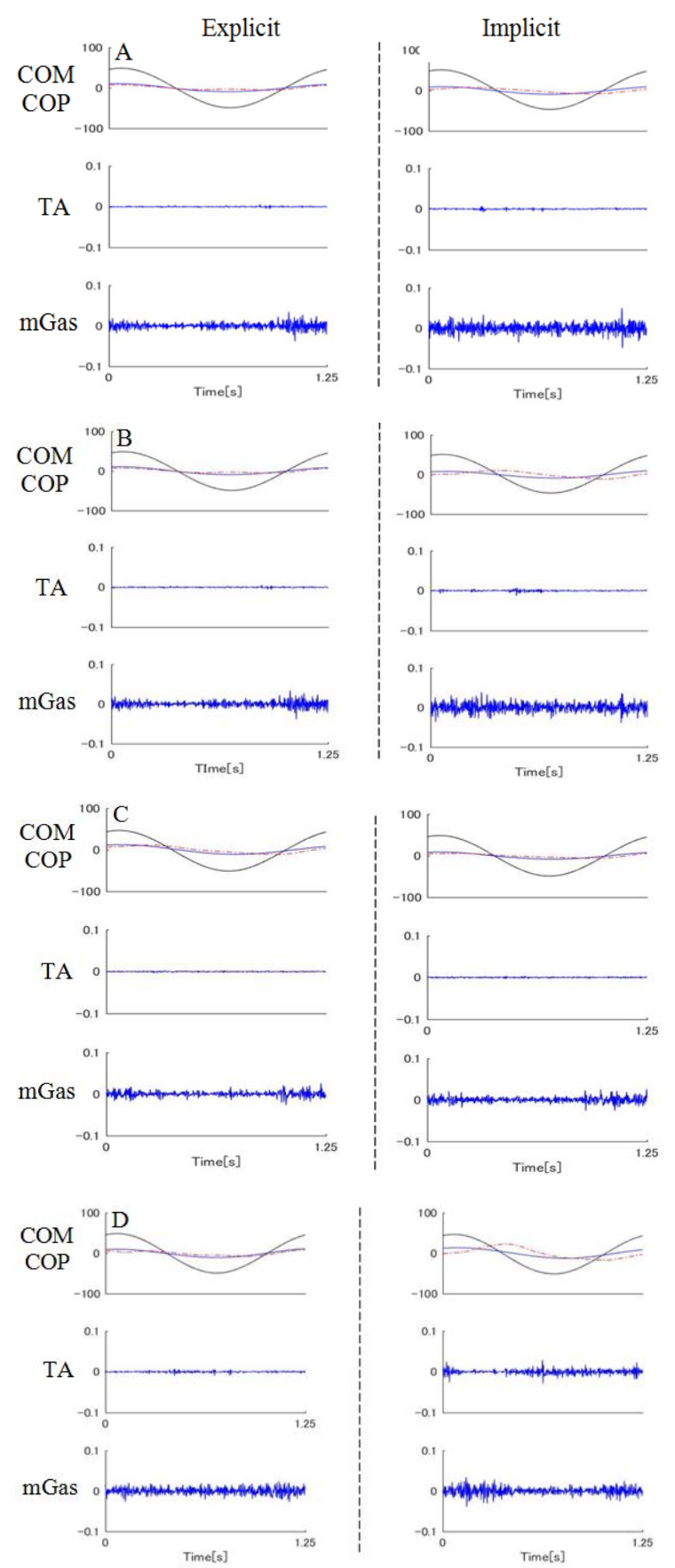

Figure 4. Motion base, COM, COP and muscle activation with the presence and absence of perception sensory (left: explicit condition, right: implicit condition, A and B: Toes Down, C and D: Toes Up, A and $\mathrm{C}$ : Eyes open, B and D: Eyes close condition).
Fig. 4 illustrates movable platform, COM, COP and muscle activation under implicit and explicit condition of perception sensory at the toes down (Fig. A and B) and toes up (Fig.4 C and D) during eyes open and eyes close. TA muscle has shown the most significant activation during toes up condition with eyes close in implicit condition. However, mGas muscle activity was lowest during toes up. Besides, during the implicit condition, mGas muscle has shown a higher activation regardless type of surface condition and visual information. On the other hand, muscle activation for TA and $\mathrm{mGas}$ have shown significant changes in implicit condition.

The mean of COM, COP, TA and mGas data measurement of all subjects was shown in Fig. 5. The black line shows the average plot for every subject. By comparing between the existence of perceiving sensation, muscle activation for TA and mGas shown a significant difference during inclined translation perturbation. Moreover, the COM and COP displacement shown a greater displacement during implicit of perception sensory condition.

Fig. 6 indicates the COM and COP displacement, TA and mGas muscle activation in response to support surface in three different conditions with different visions sensory at frequency $0.8 \mathrm{~Hz}$. All the results in kinematics and muscle activity have shown a significant difference between two different visions sensory. Meanwhile, significant increase in COM displacement was seen in all three different translation conditions, especially in the toes down condition. In terms of type of perturbation, a significant difference was observed between level and toes up condition for TA muscle. For the mGas muscle, a significant difference was observed between level and toes up condition, and between toes up and toes down condition during eyes close.

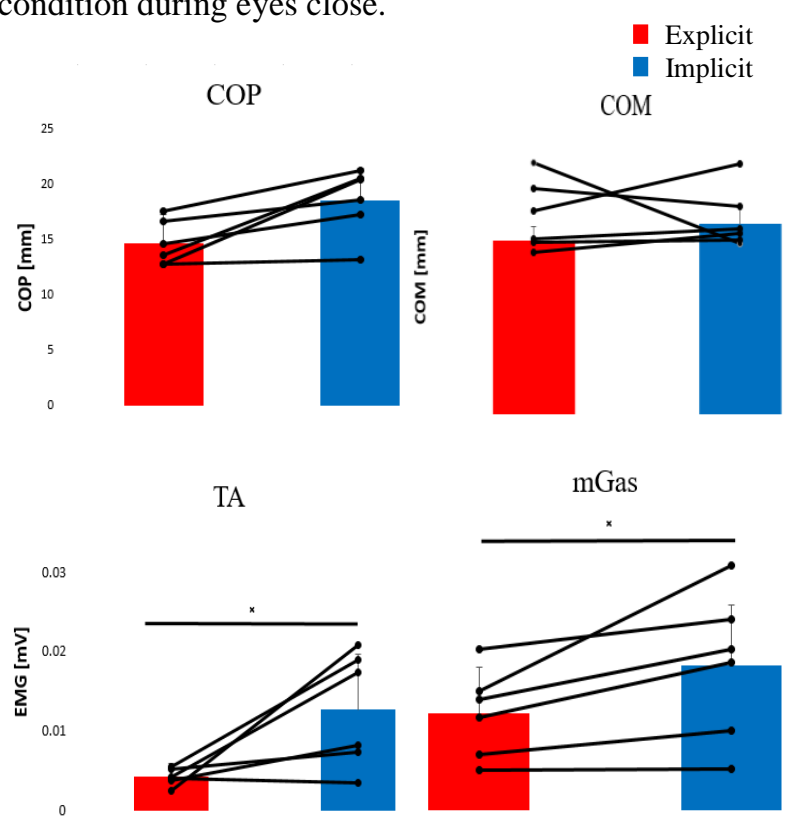

Figure 5. Mean of COM, COP,TA and $\mathrm{mGas}$ at $0.8 \mathrm{~Hz}$ during eyes close (red: explicit condition, blue: implicit condition) 

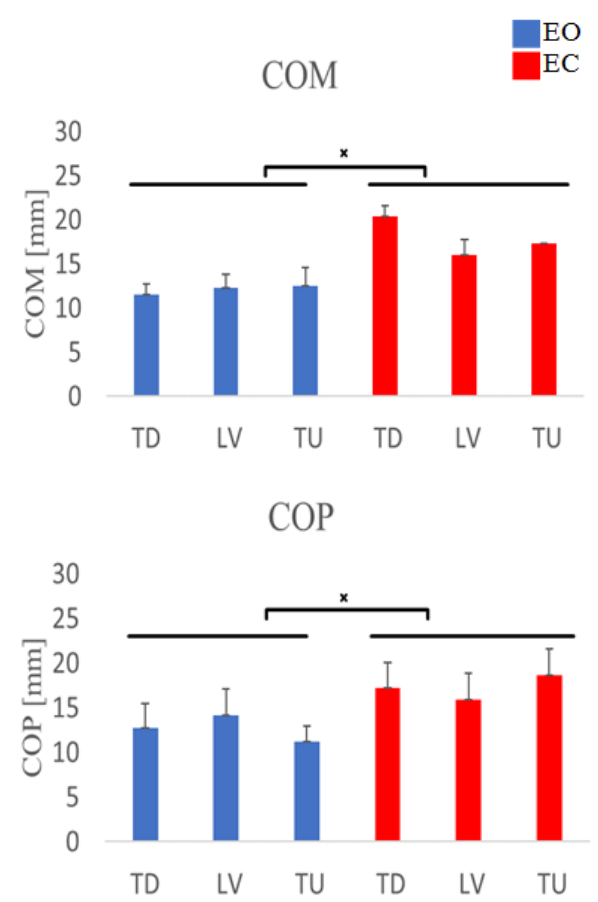

TA

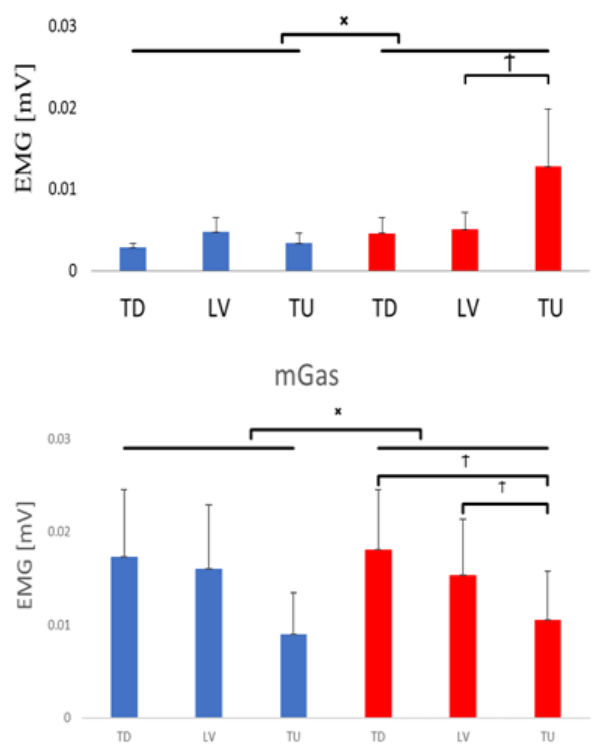

Figure 6. Group mean and standard deviation of the Center of mass (COM), center of pressure (COP) displacement and EMG data of TA and mGas muscle at $0.8 \mathrm{~Hz}$ translation frequency. The blue indicates eye open (EO) condition and the red indicates eye close (EC) condition. A significant difference between visual information is indicated by *, a significant different between the type of perturbation is indicated by $\uparrow$.

\section{DISCUSSION}

The aim of this study was to investigate whether or not the implicit inclined support surface with the changes of platform angle and frequency will influence the postural system strategy.

In the present experiment, we at first demonstrated that in the level condition, at fast perturbation translation $(0.8 \mathrm{~Hz})$, subjects tend to fix their head and trunk motion to maintain their balance. These results were agreed with
Buchanan et al. where he had suggested that by fixing the head in space, very stable platform is provided for the vestibular systems [10]. With the head fix in the space, it actually limited the sense of the Vestibulo-Ocular Reflex (VOR) which functioning for triggering eye movement to fix on a desired gaze point when the head was moving. The postural reflex on any changes due to movement can be made quickly and effectively with this mechanism. The experimental results showed that there is a relation between base support surface changes and postural system strategy (Fig. 4). In the present experiment, higher value was shown in the center of mass, center of pressure and muscle activation in implicit condition in all types of support surface changes. Here, the presence of sensation played an important role in maintaining balance. The human body still can detect the changes in support surface perturbation even the changes was unrecognizable. This can be explained by the existence of sensory receptor (muscle spindle/proprioception) which located in the belly of the muscle. Sensory receptor can detect changes in the length of the muscle and send the information that can be processed by the brain in order to determine the body position. However, mGas muscle shown low muscle activity in toes up condition. This wasagreed with Sasagawa et al. where indicated that increased passive contribution required less extensor torque generated by active muscle contraction [9].

In above results, the absence of perception sense and vision led to different center of mass and center of pressure displacement, and muscle activation value based on the type of perturbation. Furthermore, different translation support surface shown to be affected by the presence of visual information. The postural body coordination was affected by the unrecognizable support surface translation changes by applying perturbation and blocking vision information. During implicit condition along with eyes close, the body was easy to lose balance since all kinematics and muscle data were high. The weakness of vision sensory not only can lead to higher muscle activation value (Fig. 5.), it also can lead to the increased in body sway. According to Buchanan (1999), visual information helps in reducing the variability of the head's position and the position of the center of mass within the support surface defined by feet. Furthermore, visual system and vestibular system works closely to maintain balance. Therefore, the vision was proven played an important role in calibrating the vestibular systems to help optimize physical balance [13].

From this study results, standing postural strategy was effected by the type of perturbation even in implicit condition. Furthermore, the influence of the three major senses of the visual system, the vestibular system and somatosensory system that involved in posture balance control was also proven.

\section{CONCLUSION}

The aim of this paper is to investigate the perceived sensation during the support translation angle changes and influence to the postural balance strategy. The postural balance strategy was changed even in an implicit condition of perception sensory condition at inclined support surface translation. Besides, the balance becomes easy to lose under implicit of perception sensory. This study concluded that the influence from the upper center of the body due to the existence of perception sensory 
was affected the postural control strategy. Therefore, investigation in consideration of perception sensory existence is useful for fall prevention. Further study will be necessary in order to predict balancing strategy in details based on intrinsic factor such as muscle activity together with kinetic and kinematics data.

\section{ACKNOWLEDGMENT}

I would like to thank Ogawa Sho for providing data and all Posture and Gait group members for their support and advices along my $\mathrm{PhD}$ journey. Thank you.

\section{REFERENCES}

[1] D. A. Winter, "Human balance and posture control during standing and walking," Gait \& Posture, vol. 3, pp. 193-214, December 1995.

[2] N. M. Arts, "Balance disorder," NIDCD Fact Sheet, 2009.

[3] T. A. Stoffregen and L. J. Smart. Jr., "Postural instability precedes motion sickness," Brain Res Bull, vol. 47, pp. 437-448, Nov. 1998.

[4] F. B. Horak and L. M. Nashner, "Central programming of postural movements: Adaptation to altered support-surface configurations," Journal of Neurophysiology, vol. 55, no. 6, p. 1369, 1986.

[5] T. D. J. Welch and L. H. Ting, "Mechanism of motor adaptation in reactive balance control," PLos One, vol. 9, no.5, p. e96440, 2014.

[6] A. L. Adkin, J. S. Frank, M. G. Crpenter, and G. W. Oeysar, "Postural control is scaled to level of postural threat," Gait \& Posture, vol. 12, no. 2, pp. 87-93, 2000.

[7] A. D. Kuo and F. E. Zajac, "A biomechanical analysis of muscle strength as a limiting factor in standing posture," Journal of Biomechanics, vol. 26, pp. 137-150, 1993.

[8] M. Afschrift, I. Jonkers, J. De Schutter, and F. De Groote, "Mechanical effort predicts the selection of ankle over hip strategies in non-stepping postural responses," Journal of Neurophysiology, vol. 116, pp. 1937-1945, 2016.

[9] S. Sasagawa, "Balance control under different passive contributions of ankle extensors: Quiet standing on inclined surfaces," Exp. Brain Res., vol. 196, pp. 537-544, 2009.

[10] J. J. Buchanan and F. B. Horak, "Emergence of postural pattern as a vision and translation frequency," Neurophysiological, vol. 81, p.16, 1999.
[11] M. Ishikawa and S. Yamamoto, "Effect of inclined support surface on postural strategy during anterior-posterior platform translations," in Proc. IEEE EMBS, San Diego, 2012, pp. 47724775.

[12] K. Jun and S. Yamamoto, "Effect of inclined surface on postural strategy during anterior-posterior passive sway," SEATUC, 2016.

[13] J. R. Willis, S. E. Vitale, Y. Agrawal, and P. Y. Ramulu, "Visual impairment, uncorrected refractive error, and objectively measured balance in the United State," JAMA Opthamol, vol. 131, no. 8, pp. 1049-1056, 2013.

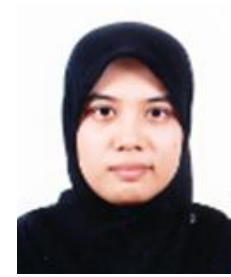

Nur Fatin Fatina bt. Mohd Ramli was born in Kelantan, Malaysia. She received Bachelor of Engineering in 2012 and Master of Engineering in 2016 from Shibaura Institute of Technology. From 2016, she admitted to Functional Control Systems at Shibaura Institute of Technology to persuade her $\mathrm{PhD}$ study. She is a member of IEEE.

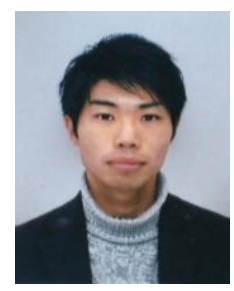

Ogawa Sho was born in Japan. He received Bachelor of Engineering in 2017 from Shibaura of Institute of Technology. Now, he is pursuing his Master in Engineering under NeuroRehabilitation Laboratory under supervision of Prof. Yamamoto Shin-ichiroh.

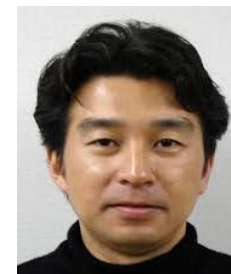

Yamamoto Shinichiroh was born in Japan. He received his Ph.D degree from the Department of Life Science at University of Tokyo in 2000.

Prof. Yamamoto is a professor at Department of Biomedical and Engineering at Shibaura Institute of Technology. His research interest including neuro-rehabilitation engineering and neural mechanism for human motor control. 